\title{
Energy Aware Routing Protocol for Cognitive Radio Networks
}

\author{
Yu Song1, Xiaoli He'2, Ralf Volker Binsack ${ }^{3}$ \\ ${ }^{1}$ Department of Network Information Management Center, Sichuan University of Science and Engineering, Zigong, China \\ ${ }^{2}$ School of Information Engineering, South West University of Science and Technology, Mianyang, China \\ ${ }^{3}$ Winterstein 14, Niddatal, Germany \\ Email: lily_820456@163.com
}

How to cite this paper: Song, Y., He, X.L. and Binsack, R.V. (2017) Energy Aware Routing Protocol for Cognitive Radio Networks. Wireless Sensor Network, 9, 103115.

https://doi.org/10.4236/wsn.2017.93006

Received: March 6, 2017

Accepted: March 28, 2017

Published: March 31, 2017

Copyright (c) 2017 by authors and Scientific Research Publishing Inc. This work is licensed under the Creative Commons Attribution International License (CC BY 4.0).

http://creativecommons.org/licenses/by/4.0/

\section{cc) (i) Open Access}

\begin{abstract}
In this article, a routing protocol EARP (Energy Aware Routing Protocol) with the terminal node is proposed, to deal with the impact of the limited energy resources of Cognitive Radio Networks on the whole network routing. The protocol allows choosing the route from the neighbor nodes in different transmission paths, according to energy consumption of a single node and the full path. If the path breaks, the protocol will increase local routing maintenance strategy. It effectively reduces the retransmission caused by the situation, and improves the routing efficiency. It also can prevent the link transmission process selecting the fault route due to the energy depletion. Through simulation experiments compared with the LEACH (Low Energy Adaptive Clustering Hierarchy) routing protocol, the results showed that in the same experimental environment, the proposed EARP could obviously balance the load, protect low energy nodes, prolong the network survival time and reduce packet loss rate and packet delay of data delivery. So it can improve the energy consumption of sensing node and provide routing capabilities.
\end{abstract}

\section{Keywords}

Energy Aware Routing Protocol, Route Strategy, Route Maintenance Strategy Network Lifetime, Packet Loss Rate, Relative Residual Energy

\section{Introduction}

Haykin [1] has precisely defined Cognitive Radio (CN) in the following way: "A cognitive radio transmitter will learn from the environment and adapt its internal states to statistical variations in the existing radio frequency (RF) stimuli by adjusting the transmission parameters (e.g., frequency band, modulation mode, and transmission power) in real-time and on-line manner". A cognitive radio 
network consists of many mobile nodes. The terminal node generally uses the battery to supply power to ensure its long distance transmission characteristics. Therefore, energy resources are usually used as an important part in cognitive radio networks. Energy depletion of one node in the network means that the affected node cannot further participate in subsequent data delivery, and thus is named dead node. A dead node will cause a series of problems such as link fault, data interrupt and excessive energy consumption, etc. In traditional wireless sensor networks, routing protocol is only concerned with the efficiency, and the minimum hop count as the selection criterion of the routing protocol [2]. It makes the center position of the nodes in the network as a link forwarding node, thus its energy exhaustion due to selection as the link cluster head, will result in congestion of the data points, and the loss of large amounts of data and retransmission. However, it can be seen that a routing protocol which ignores the node energy factor incurs high cost and high energy consumption [3].

Nowadays, the energy saving algorithm based on node energy consumption has attracted much attention of researchers. Huang et al. summed up the characteristics of different routing protocols in following: a sensor network of clusters with a hierarchical routing protocol to increase network lifetime was reported [4]. They showed, with many sensor nodes, reduction of energy consumption by hierarchical routing instead of flat routing. However, their work is not related to mobile CRNs. Heinzelman et al. proposed an energy-efficient routing protocol with low end-to-end delay, e.g., low energy adaptive clustering hierarchy (LEACH) [5]. However, the LEACH protocol did not consider the energy state of cluster heads and sensor nodes. To enhance the energy efficiency of a sensor network, various approaches have been made. In case of wide band sensors such as orthogonal frequency division multiplexing (OFDM) type sensors, peak power reduction is critical [6] [7]. Optimal sleep-wake scheduling to extend the network lifetime was investigated by Deng et al. [8]. However, these schemes result in an increment of the packet delay as each sensor node waits for its next hop relay to wake up. Kim et al. proposed packet forwarding by each sensor node to the first awake neighbor node [9]. This method is prone to worsening the packet delay even more if the first awake node is in a direction opposite to the sink or destination node, Deng et al. [8].

Based on the above analysis, this paper proposes a new energy aware routing protocol (EARP) for the CRNs. According to the routing strategy, this protocol can achieve the goal of improving network performance at different energy stages (the normal stage, the warning stage, the danger stage) [10]. In this paper, the concept of relative residual energy of the node is introduced as the basis for calculating the cost of routing. The route discovery strategy is adopted to collect the energy information of nodes themselves and other nodes in the link. First, the node energy consumption and residual energy model are established. Secondly, the network lifetime is used as the first measurement criterion in the evaluation of the simulation performance. Therefore, it is helpful to analyze the performance of the protocol to establish the multi scale network lifetime model [11]. Comparing 
with the LEACH protocol, the EARP has some advantages, such as relatively small energy consumption, balancing the load, protecting low energy nodes, prolonging the network survival time and reducing packet loss rate and packet delay of data delivery. This is mainly because the EARP has chosen the neighbor sensing node with the maximum residual energy as the next node hop, establishing the data forward routing with the minimum energy consumption, thus ensuring the energy balance of CRNs.

The rest of this paper is organized as follows. The energy-consuming model of CRNs is reviewed in Section 2. Section 3 describes the routing protocol based on residual energy aware. Then Section 4 provides the result of simulation experiment. Finally, Section 5 concludes this paper and outlines the future work of node energy consumption.

\section{Energy-Consuming Model of CRNS}

In the cognitive radio networks, nodes may need to communication with the other nodes which are beyond their ranges. As shown in Figure 1, if the source node transmits signal to the destination node that beyond its rang, it usually uses its neighbor node to receive and repeat the signal. The neighbor node is called a repeater node. Considering the different node position, the routing process may require multiple nodes forwarding or resending data to complete the transfer [12]. This end-to-end transmission process is shown in Figure 1.

\subsection{Energy Consumption and Residual Energy Model}

According to the network model of W. B. Heinzelman [5] and the LEACH (Low Energy Adaptive Clustering Hierarchy) protocol, the energy consumption model of sensing node in communication process is shown in Figure 2.

Assuming that the distance between a pair of transmitting and receiving node is $d$, in the free space propagation, the receiving node needed energy to receive the signal is $E_{\min }$. Energy consumption of the amplifier is $E_{\text {amp }}$, which is shown in Equation (1):

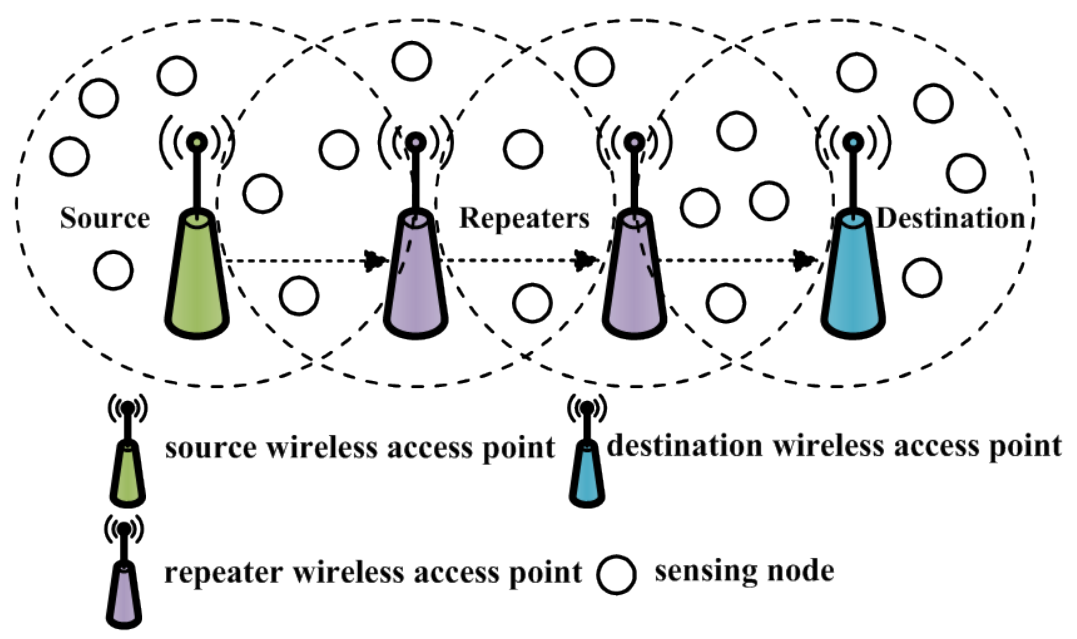

Figure 1. End-to-end transmission of CRNs. 


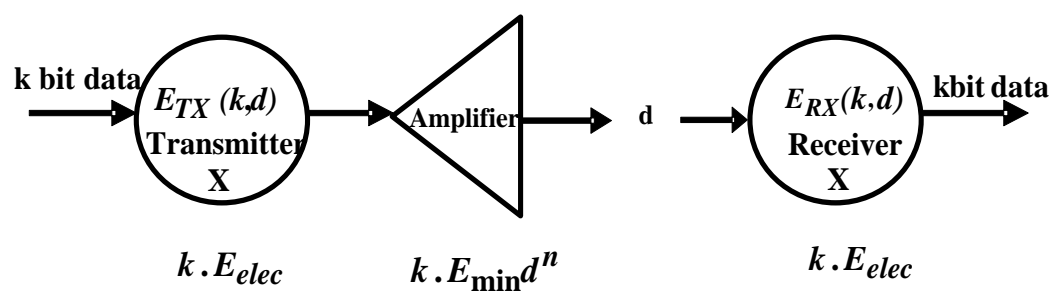

Figure 2. Energy consumption model of sensing node.

$$
E_{\text {amp }}(d)=k d^{n} E_{\min } \text {. }
$$

In Equation (1), $n$ value generally equals 2 or 4 , and $k$ is a constant. It indicates that the receiving signal energy is weakened with the increase of the node distance. In this paper, $n$ equals 2 and $k$ equals 1 . So it can be simplified as Equation (2).

$$
E_{\text {amp }}(d)=d^{2} E_{\min } \text {. }
$$

Supposing that $E_{\text {elec }}$ is the energy consumption of the terminal node in the process of transmitting and receiving, the energy transfer parameter is $E_{T X}$, and the receiving parameter is $E_{R X}$. For a pair of transmitting and receiving nodes, $l$ bit data is sent and received, and the energy computational equation $E_{T R}$ is showed in Equation (5):

$$
\begin{gathered}
E_{T X}(l, d)=l\left(E_{\text {elec }}+d^{2} E_{\text {min }}\right), \\
E_{R X}(l)=l E_{\text {elec }}, \\
E_{T R}(l, d)=E_{T X}(l, d)+E_{R X}(l)=2 l E_{\text {elec }}+l d^{2} E_{\text {min }} .
\end{gathered}
$$

For a single node $i, E_{i}^{o}$ is the initial energy value, $E_{i}^{c}$ is the consumption energy, $E_{i}^{c r}$ is the current residual energy value, and $E_{i}^{r}$ is relative residual energy value, $R$ is the residual energy percentage.

$$
\begin{gathered}
E_{i}^{c r}=E_{i}^{o}-E_{i}^{c}, \\
E_{i}^{r}=E_{i}^{c r}-E_{i-1}^{c r}, \\
R=\frac{E_{i}^{c r}}{E_{i}^{o}} .
\end{gathered}
$$

In a complete path of data transmission, the link includes the source node, the destination node and all the intermediate nodes, $E_{R}$ is the relative residual energy value of all nodes. $N$ is the sum of the nodes in the path.

$$
E_{R}=\sum_{i=1}^{N} E_{i}^{r}=\sum_{i=1}^{N} E_{i}^{c r}-E_{i-1}^{c r} .
$$

In Figure 3, the link is composed of five nodes, which is 1-2-3-4-5. Figure 3 expresses that the sum energy of nodes is same, but the energy value of each node is different. Network fairness and load balance can affect the rate of energy consumption of nodes, which can result in the difference of the residual energy value of the nodes in a certain link or region. If the sum of link energy is same, the link of Figure 3(b) is more stable than Figure 3(c), the network lifetime of 


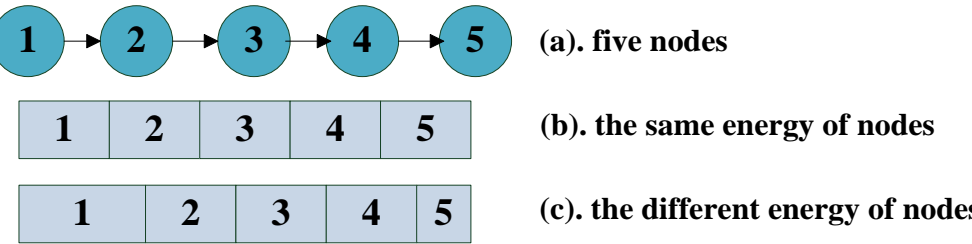

Figure 3. Different node residual energy values.

Figure 3(b) is better than Figure 3(c). In Figure 3(c), the node with large residual energy value can cover the node with small residual energy value. The node with too large or too small residual energy value is called a singular point, which are the unstable factors of the link. So the node 1 and 5 are the singular points.

Due to the network lifetime of the routing protocol as the function standard in this paper, if the residual energy of each node is the same as link routing cost influence degree, then they have the same weight, the singular point will affect the optimal routing of the link effect.

\subsection{Network Lifetime Model}

Presuming that the data transmission network is reversible, so it does not cause a one-way link. Thus only node $i$ sending data can cause the node $j$ energy consumption. $E_{i}^{o}$ is the initial energy value of $i$ node. The transmission energy consumed by the $M_{i}$ unit information is $E_{T X}\left(M_{i}, d\right)$, and the receiving energy is $E_{R X}\left(M_{i}\right)$. When the node $i$ transmitting $M_{1}$ units and receiving $M_{2}$ units needs to consume energy, the energy value $E_{i}^{w}$ is Equation (10).

$$
E_{i}^{w}=E_{T X}\left(M_{i}, d\right)+E_{R X}\left(M_{i}\right)=E_{T X}\left(M_{1}, d\right)+E_{R X}\left(M_{2}\right) .
$$

If $E_{i}^{w}=E_{i}^{o}$, the energy of $i$ node is depleted. $T_{i}$ is the network lifetime of $i$ node. $T_{\text {nek }}$ is the whole network lifetime. $T_{s}(i)$ is the time of the $i$ node be sent. $T_{r}(i)$ is the time of the node $i$ arriving. $T_{i} \in\left(T_{1}, T_{2} \cdots T_{N}\right)$

$$
T_{\text {nek }}=\frac{1}{N} \sum_{i=1}^{N}\left[T_{r}(i)-T_{s}(i)\right] \text {. }
$$

\section{Routing Protocol Based on Residual Energy Aware}

\subsection{Route Strategy}

If the overall residual energy value $E_{R}$ of the link is greater, then the link is freer. After selecting this link as the routing path, it can play an important role in balancing the network load. At this point, the route choice should avoid these singular points, as far as possible to save the residual energy to prolong the network lifetime. If the nodes' residual energy is in the stage of danger, they are not assumed to be forwarded. Only the source node and the destination node are used to prolong the survival time.

In this paper, two threshold $R_{1}$ and $R_{2}$ are set in the relative residual energy value of the node, and the node energy is divided into three stages: the normal stage (the parameter is $R_{s 1}$ ), the warning stage (the parameter is $R_{s 2}$ ) and the danger stage(the parameter is $R_{s 3}$ ). $R_{1}$ is set to smaller than $R_{2}$ commonly. 
According to Equation (8), the residual energy percentage $R$ will compared with $R_{1}$ and $R_{2}$.

So according to the route strategy analysis, when the residual energy is in the normal stage, the relative residual energy value of the node $E_{r}$ is high.

$$
R_{\mathrm{s} 1}=\text { Normal } \quad R_{2}<R<1.0 .
$$

When the residual energy is in the warning stage, the value of $E_{r}$ is middle.

$$
R_{s 2}=\text { Warning } \quad R_{1}<R<R_{2} \text {. }
$$

When the residual energy is in danger stage, the node is protected and not forwarded. Then the value of $E_{r}$ is low.

$$
R_{\mathrm{s} 3}=\text { Danger } \quad 0.0<R<R_{1} .
$$

\subsection{Route Request}

If the node wants to send data, it first finds out whether there is a valid route available in the routing table [13]. It needs to send a Route Request (RREQ) message to its neighbor node. RREQ message includes source ID, destination ID, sequence number, the residual energy message $E_{R}$ and the relative residual energy value $E_{r}$ as is shown in Figure 4. The format of the RREQ message is given as following:

When the neighbor nodes and intermediate nodes receive the RREQ message from the source node, they first check whether their route in the routing table is effective according to the requirements, then avoid the loop generating sequence [14] [15] [16]. If there is not an effective route to the destination node, they continue forwarding the work. The forwarding node obtains the energy information $E_{R}$ and $E_{r}$ from the format of the RREQ message. Comparing with their own energy information, they decide whether they need to update the routing table and the energy information of RREQ. The relationship of $E_{r}(i), E_{r}($ old $)$ and $E_{r}$ (new) is the following:

$$
\text { If } E_{r}(i)<E_{r} \text { (old) }
$$

then

$$
E_{r}(\text { new })=E_{r}(i),
$$

else

$$
E_{r}(\text { new })=E_{r}(\text { old }),
$$

the new residual energy $E_{R}$ (new) value is following:

\begin{tabular}{|l|l|l|l|l|}
\hline Source ID & Destination ID & $\begin{array}{l}\text { Sequence } \\
\text { Number }\end{array}$ & $E_{R}$ & $E_{r}$ \\
\hline
\end{tabular}

(a).format of the RREQ message

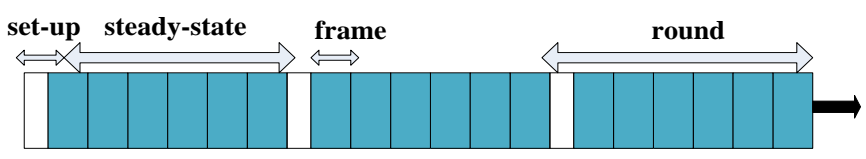

(b). time sequence diagram of EARP

Figure 4. Format of the EARP. 


$$
E_{R}(\text { new })=E_{r}(\text { old }) \sum_{i=1}^{N} E_{r}(i) .
$$

\subsection{Route Discovery}

When the route request reaches the destination node, the destination node will start the timer $T$ to set the delay for receiving the valid routing request. If the intermediate node contains the destination node, the RREQ can be sent directly to the source node for completing the route discovery process. The destination node receives the RREQ, comparing the energy information $E_{r}$ with residual energy threshold $R_{1}$ and $R_{2}$. According to Equations ((11) to (13)), it selects the routing strategy of current link node energy state. After the delay timer is $T$, the RREQ is sent to the source node to complete the route discovery process [14].

\subsection{Route Establishment}

In the working process of CRNs, if the current node residual energy has been run out, then the sensing node needs to choose the next-hop routing sensing node. As specific selection is shown in Figure 5, the specific thoughts are:

Step 1: The node of the largest residual energy is selected from neighbor sensing node.

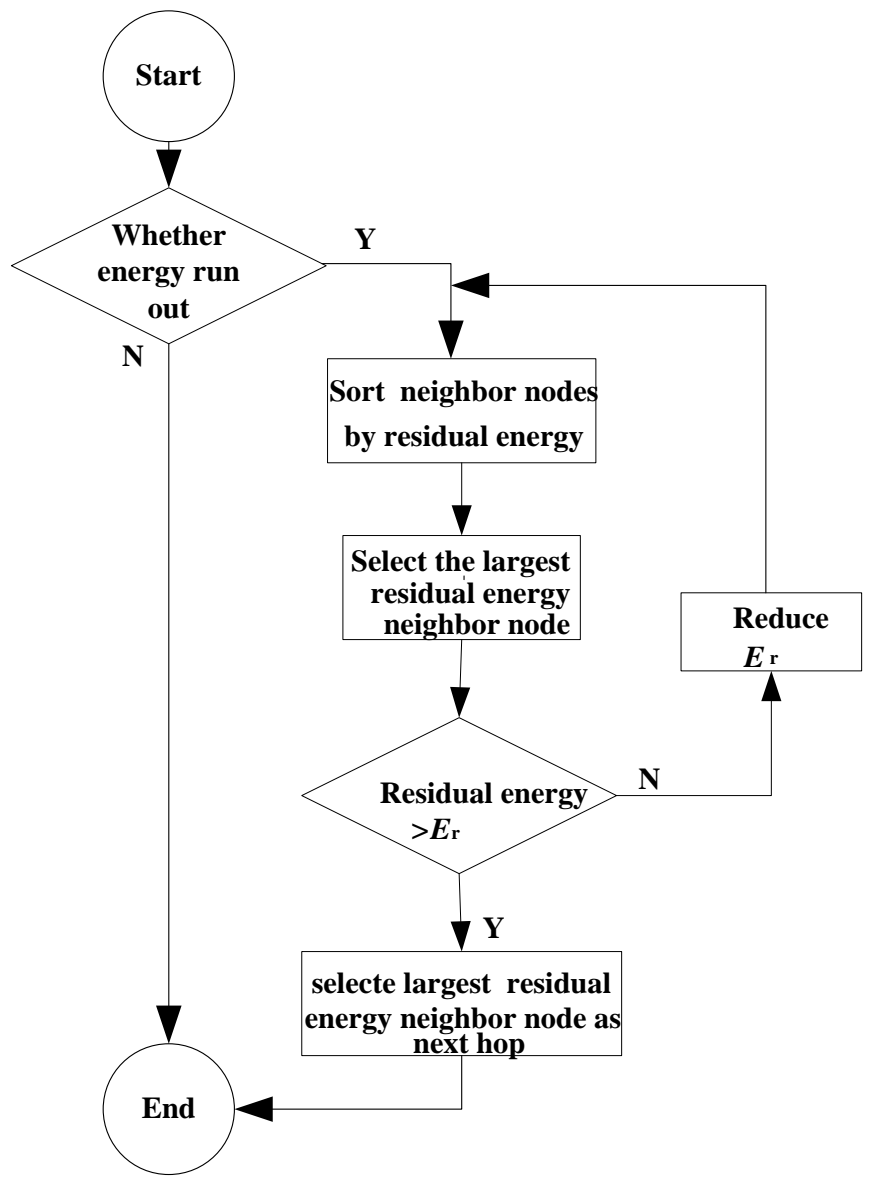

Figure 5. Building process of data routing. 


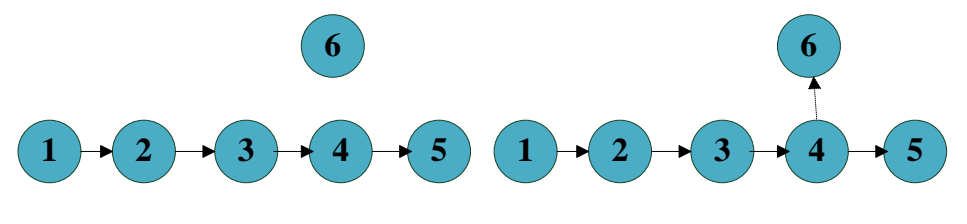

(a) initial link

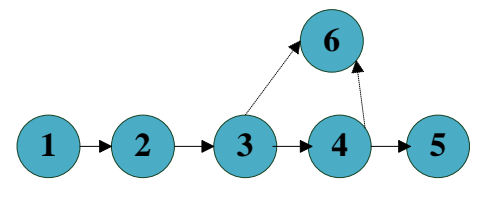

(c) local request (b) local sponsor

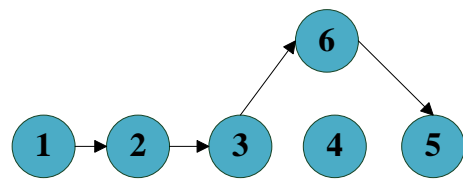

(d) new route setup

Figure 6. Local route maintenance strategy.

Step 2: If the residual energy of selected neighbor sensing node is greater than predetermined threshold $\left(E_{r}\right)$, then the neighbor sensing node is taken as the next hop of sensing nodes of transmitting data.

Step 3: If the residual energy of selected neighbor sensing node is less than predetermined threshold $\left(E_{r}\right)$, then the value of $E_{r}$ is decreased to re-select the next hop node.

\subsection{Route Maintenance Strategy}

When the residual energy of the node is reduced to the trigger that power saving protection mechanism, the link will be broken, and lead to the reselecting alternative way of data transmission. According to the EARP, the residual energy of the node can be predicted in advance [17]. Nodes predict that the link will be broken due to the shortage of the residual energy, which will trigger local route maintenance strategy to avoid the fault of the route [18] [19]. Especially in the later stage of the network, most of the nodes have their own energy consumption, which is more likely to happen.

In Figure 6, the node 1-2-3-4-5 forms a complete transmission link, assuming that there is no one-way link in the network. In the process of data forwarding, the node 4 is unable to continue transmitting the data as its own residual energy value is too low. The EARP predicts that the energy is too low in advance and starts the local maintenance strategy. Node 4 sends RREQ information to its surrounding nodes, and its neighbor node 6 receives information. Node 6 finds its routing table can be used to replace the node 4 routing, and checks its own residual energy value. If the value of the node 6 is in conformity with the forwarding condition, it returns information to the node 4 , and informs the node 4 of the upstream node 3 , then the node 4 enters the wait state. This process is shown in Figure $6(\mathrm{c})$. Node 3 completes the work of routing selection, which includes the energy sufficient of link data transmission. It effectively avoids the routing data due to lack of the residual energy retransmitting, which is shown in Figure 6(d). Finally the new route setup is 1-2-3-6-5.

According to local route maintenance strategy, it can design the algorithm of EARP. 


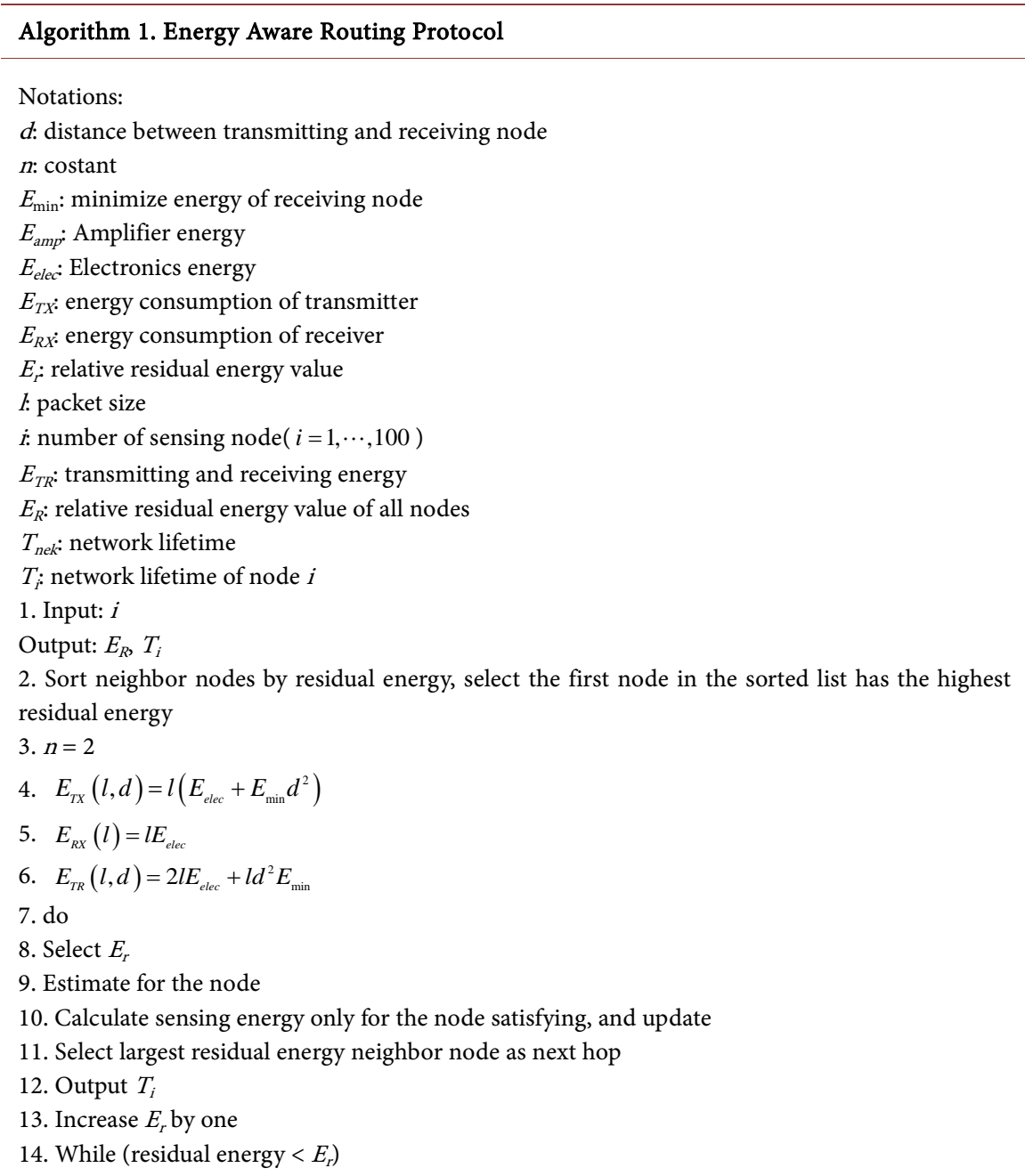

\section{Simulation Experiment}

\subsection{Simulation Environment}

In order to test the effectiveness and superiority of energy aware routing protocol for Cognitive Radio Networks, simulation experiment is achieved using Matlab 2015b simulation software in the computer of AMD dual-core $2.20 \mathrm{GHz}, 4 \mathrm{G}$ RAM, Windows 7 operating system. The simulation results of the protocol EARP in this paper are comparable and convincing. Comparing with the LEACH protocol, it makes a comprehensive analysis of their average energy consumption, the network lifetime, packet loss rate, etc. The simulation parameters are shown in Table 1.

\subsection{Result and Analysis}

1) Comparison of average energy consumption of nodes

Generally along with increased production cycle of time sequence, the energy consumption of all routing protocols for CRNs has been declining. It can be clearly seen from Figure 7, comparing with the LEACH protocol, the EARP has relatively small energy consumption, this is mainly because the EARP has reduced 
Table 1. Common simulation parameters.

\begin{tabular}{cccc}
\hline Parameter name & Value & Parameter name & Value \\
\hline Area size & $200 \mathrm{~m} \times 200 \mathrm{~m}$ & Request packet & $50 \mathrm{bit}$ \\
The total number of nodes & 100 & Transmission packet & $40 \mathrm{bit}$ \\
Packet size 1 & $4000 \mathrm{bit}$ & $d$ & $50 \mathrm{~m}$ \\
Sensing node & $(0,0)$ & $E_{\text {amp }}$ & $0.0013 \mathrm{pJ} / \mathrm{bit} / \mathrm{m}^{2}$ \\
Cluster radius & $20 \mathrm{~m}$ & $E_{\text {elec }}$ & $50 \mathrm{~nJ} / \mathrm{bit}$ \\
$E_{T R}$ & $4 \times 10^{-3} \mathrm{~J} / \mathrm{s}$ & $E_{\min }$ & $2 \times 10^{-3} \mathrm{~J} / \mathrm{s}$ \\
$E_{r}$ & $4.5 \times 10^{-5} \mathrm{~J} / \mathrm{s}$ & $T_{1}$ & $1 \mathrm{~ms}$ \\
$E_{R}$ & $9 \times 10^{-4} \mathrm{~J} / \mathrm{s}$ & $E_{i}^{o}$ & $0.1 \mathrm{~J}$ \\
\hline
\end{tabular}

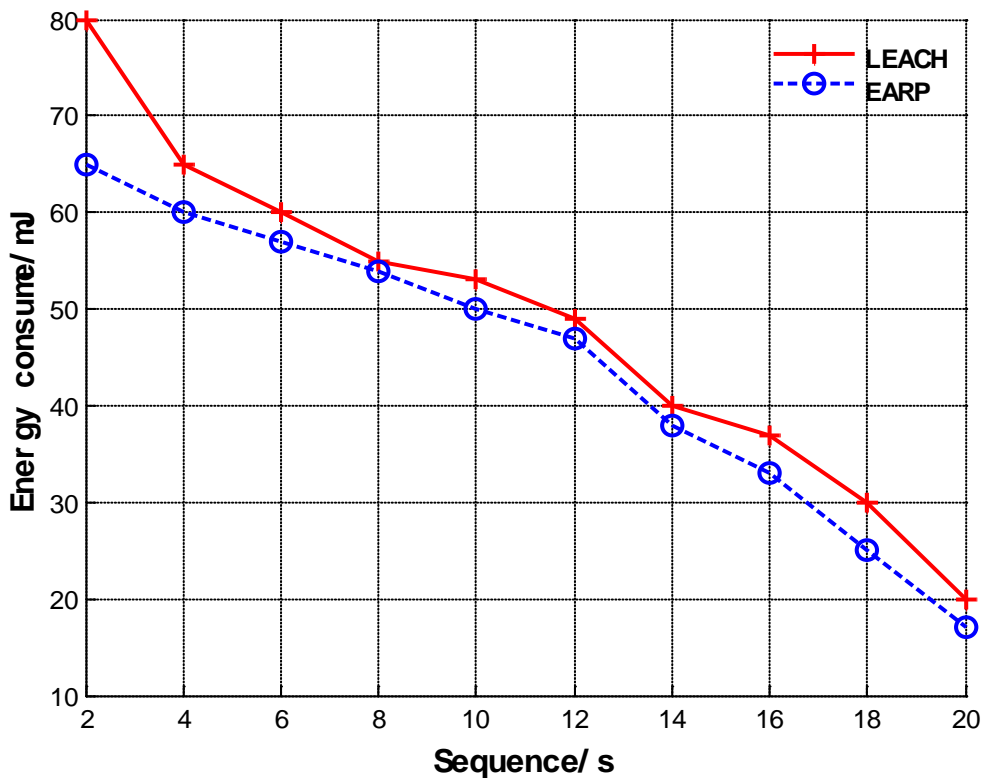

Figure 7. Comparison of average energy consumption of nodes.

the energy consumption of continuous transmission. It has chosen the neighbor sensing node with the maximum residual energy as the next node hop, establishing the data forward routing with the minimum energy consumption, thus ensuring the energy balance of the entire CRNs. Because of the energy consumption balance, the two protocols become close to each other at the time sequence between 8 and 12 from the Figure 7 .

2) Comparison of network lifetime

Network lifetime is usually described using survival of network of sensing nodes. The survival curve of sensing nodes for protocol is shown in Figure 8. As can be seen from Figure 8, the two network routing protocols are decreased continuously with the network node survival of network working hours. But at the same time point, the numbers of live nodes of the EARP are more than that of the LEACH.

3) Comparison of packet loss rate

Packet loss rate is a meaningful indicator of measuring routing protocol per- 


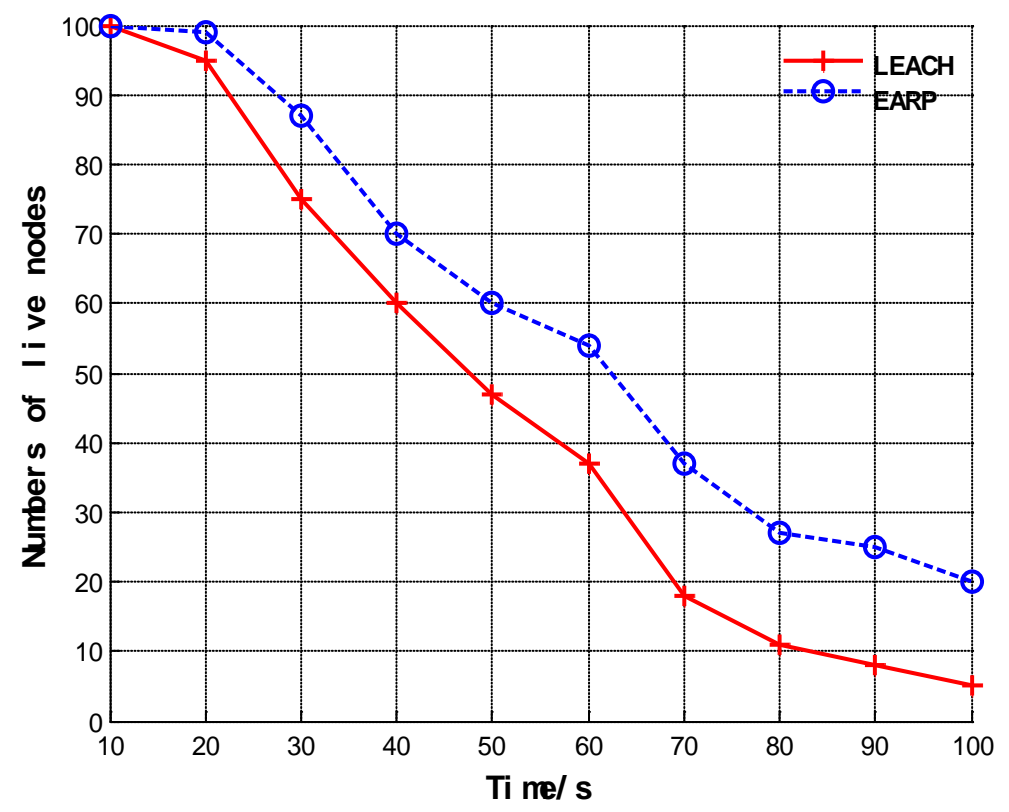

Figure 8. Comparison of network lifetime.

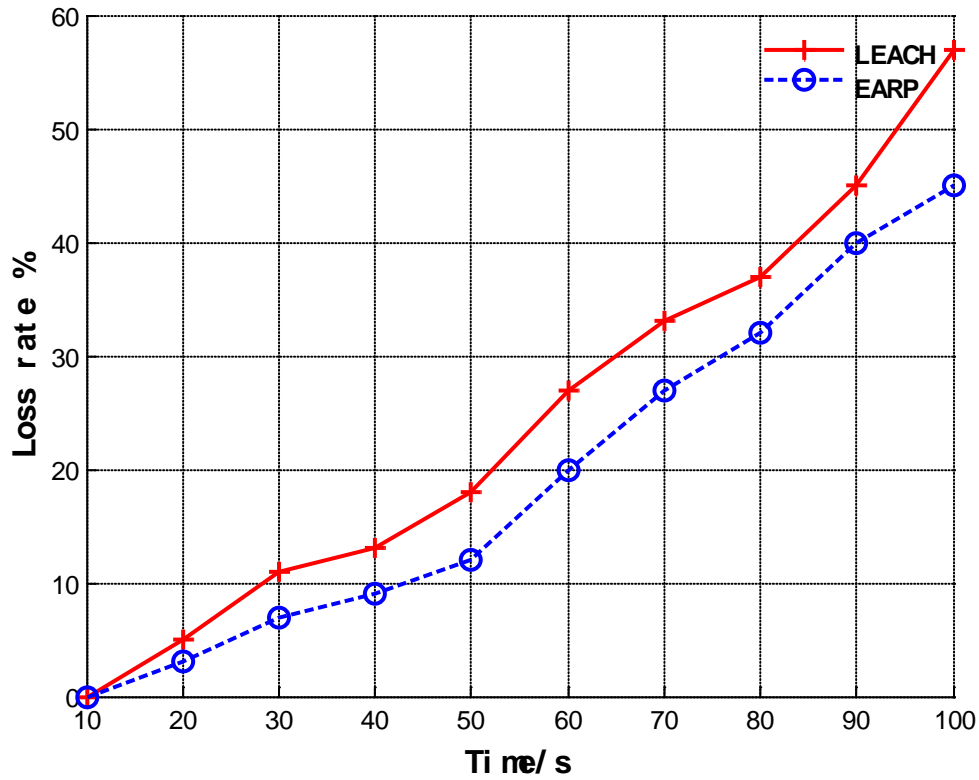

Figure 9. Comparison of packet loss rate.

formance for CRNs. The packet loss rate curve of CRNs for this protocol is shown in Figure 9. As can be seen from Figure 9, with the increased run time of CRNs, the packet loss rate of sensing is rising, which is mainly because as the network time increases, the death rate of network node is increased. If the death of sensing node in data forwarding routing occurs, it may lead to failure in the established data forwarding routing. The sensing nodes no longer play the role of relaying the data, and the packet on the route would be lost. At the same time, comparing with the LEACH, the EARP in this paper has smaller packet loss rate, which is mainly because the routing protocol in this paper has introduced the energy aware mechanism, saving energy consumption, and ensuring the com- 
munication quality of the network, thereby improving the success rate of packet forwarding.

\section{Conclusion}

In this paper, it has been proposed an energy aware routing protocol for CRNs, named EARP. The proposed protocol balances the traffic load among different CRNs nodes according to their nodal residual energy and prolongs the lifetime of individual CRNs node as well as the overall networks. Extensive simulation results have shown that the proposed protocol can decrease the message delay and the consumed energy. In addition, it increases the system throughput, the routing success rate, the ratio of survival nodes and the network lifetime. Accordingly, the routing path formed by EARP is more reliable and stable. Future works should focus on the theoretical performance analysis of the algorithm. In summary, the proposed energy aware routing scheme provides an efficient and practical solution for data routing in cognitive radio networks.

\section{Acknowledgements}

This work was supported in part by: National Natural Science Foundation of China, No. 61379005; 2016 Key Base of Tourism and Scientific Research of Sichuan Provincial Tourism Administration, No. ZHZ16-02;2014 Key Laboratory of Higher Education of Sichuan Province for Enterprise Informationalization and Internet of Things, No. 2014WYY03; 2014 Artificial Intelligence Key Laboratory of Sichuan Province, No. 2014RYY02; 2015 Key Base of Tourism and Scientific Research of Sichuan Provincial Tourism Administration, No. ZHY15-04; 2014 Key Laboratory of Higher Education of Sichuan Province for Enterprise Informationalization and Internet of Things, No. 2014WYY02.

\section{References}

[1] Haykin, S. (2005) Cognitive Radio: Brain-Empowered Wireless Communications. IEEE Journal on Selected Areas in Communications, 23, 201-220. https://doi.org/10.1109/JSAC.2004.839380

[2] Zheng, S., Wu, W., Zhang, Q. and Zhang, N. (2012) Routing Protocol Base on Energy Aware in Ad Hoc Networks. Journal Communications, 33, 9-16.

[3] Usman, M., Har, D. and Koo, I. (2016) Energy-Efficient Infrastructure Sensor Network for Ad Hoc Cognitive Radio Network. IEEE Sensors Journal, 16, 2775-2787. https://doi.org/10.1109/JSEN.2016.2516018

[4] Huang, Z., Cheng, Y. and Liu, W. (2011) A Novel Energy-Efficient Routing Algorithm in Multi-sink Wireless Sensor Networks. 2011 IEEE 10 th International Conference on Trust, Security and Privacy in Computing and Communications, Changsha, 16-18 November 2011, 1646-1651. https://doi.org/10.1109/trustcom.2011.228

[5] Heinzelman, W.B., Chandrakasan, A.P. and Balakrishnan, H. (2002) An Application-Specific Protocol Architecture for Wireless Microsensor Networks. IEEE Transactions on Wireless Communications, 1, 660-670. https://doi.org/10.1109/TWC.2002.804190

[6] Hong, E., Kim, H., Yang, K. and Har, D. (2013) Pilot-Aided Side Information Detection in SLM-Based OFDM Systems. IEEE Transactions on Wireless Communica- 
tions, 12, 3140-3147. https://doi.org/10.1109/TWC.2013.061713.120280

[7] Hong, E., Min, S. and Har, D. (2010) SLM-Based OFDM System without Side Information for Data Recovery. Electronics Letters, 46, 255-256.

https://doi.org/10.1049/el.2010.3323

[8] Deng, R., Chen, J., Yuen, C., Cheng, P. and Sun, Y. (2012) Energy-Efficient Cooperative Spectrum Sensing by Optimal Scheduling in Sensor-Aided Cognitive Radio Networks. IEEE Transactions on Vehicular Technology, 61, 716-725. https://doi.org/10.1109/TVT.2011.2179323

[9] Kim, J., Lin, X., Shroff, N.B. and Sinha, P. (2010) Minimizing Delay and Maximizing Lifetime for Wireless Sensor Networks with Anycast. IEEE/ACM Transactions on Networking (TON), 18, 515-528.

[10] Tabassum, M., et al. (2016) An Energy Aware Event-Driven Routing Protocol for Cognitive Radio Sensor Networks. Wireless Networks, 22, 1523-1536. https://doi.org/10.1007/s11276-015-1043-8

[11] Kanakala, S., Ananthula, V.R. and Vempaty, P. (2014) Energy-Efficient Cluster Based Routing Protocol in Mobile Ad Hoc Networks Using Network Coding. Journal of Computer Networks \& Communications, 2014, Article ID: 351020.

[12] Lu, Y.M. and Wong, V.W.S. (2007) An Energy-Efficient Multipath Routing Protocol for Wireless Sensor Networks. International Journal of Communication Systems, 20, 747-766. https://doi.org/10.1002/dac.843

[13] He, X., Song, Y. and Binsack, R.V. (2016) Energy-Aware-Based Routing Protocol for Wireless Sensor Network. International Journal of Simulation: Systems, Science and Technology, 17, 5.1-5.5.

[14] Sarma, H.K.D., Bhuyan, B. and Dutta, N. (2013) An Energy Balanced Routing Protocol for Cognitive Wireless Sensor Networks. Proceedings of the World Congress on Engineering and Computer Science, San Francisco, 23-25 October 2013.

[15] Shah, G.A. and Akan, O.B. (2013) Spectrum-Aware Cluster-Based Routing for Cognitive Radio Sensor Networks. 2013 IEEE International Conference on Communications (ICC), Budapest, 9-13 June 2013, 2885-2889.

[16] Oey, C.H.W., Christian, I. and Moh, S. (2012) Energy- and Cognitive-Radio-Aware Routing in Cognitive Radio Sensor Networks. International Journal of Distributed Sensor Networks, 2012, Article ID: 636723. https://doi.org/10.1155/2012/636723

[17] Heinzelman, W.B., Chandrakasan, A.P. and Balakrishnan, H. (2002) An Application-Specific Protocol Architecture for Wireless Microsensore Networks. IEEE Transactions on Wireless Communications, 1, 660-670

[18] Kamruzzaman, S.M. (2012) Energy-Aware Routing Protocol for Cognitive Radio ad Hoc Networks. IET Communications, 6, 2159-2168. https://doi.org/10.1049/iet-com.2011.0698

[19] Li, X. and Zekavat, S.A. (2010) Spectrum Sharing across Multiple Service Providers via Cognitive Radio Nodes. IET Communications, 4, 551-561.

https://doi.org/10.1049/iet-com.2009.0113 
Submit or recommend next manuscript to SCIRP and we will provide best service for you:

Accepting pre-submission inquiries through Email, Facebook, LinkedIn, Twitter, etc. A wide selection of journals (inclusive of 9 subjects, more than 200 journals)

Providing 24-hour high-quality service

User-friendly online submission system

Fair and swift peer-review system

Efficient typesetting and proofreading procedure

Display of the result of downloads and visits, as well as the number of cited articles Maximum dissemination of your research work

Submit your manuscript at: http://papersubmission.scirp.org/

Or contact wsn@scirp.org 